\title{
A novel etiology for craniospinal hypovolemia: a case of inferior vena cava obstruction
}

\author{
Neeraj Kumar, MD, ${ }^{1}$ Newton B. Neidert, MD, ${ }^{2}$ Felix E. Diehn, MD, ${ }^{3}$ Norbert G. Campeau, MD, ${ }^{3}$ \\ Jonathan M. Morris, MD, ${ }^{3}$ and Haraldur Bjarnason, MD ${ }^{2}$ \\ Departments of ${ }^{1}$ Neurology, ${ }^{2}$ Radiology_Vascular and Interventional Radiology, and ${ }^{3}$ Radiology_Neuroradiology, Mayo Clinic, \\ Rochester, Minnesota
}

\begin{abstract}
The authors report on a patient with craniospinal hypovolemia and inferior vena cava obstruction, and describe how the two conditions may be linked. This unique report further advances the emerging literature on spinal CSF venous fistulae. https://thejns.org/doi/abs/10.3171/2018.2.SPINE171373
\end{abstract}

KEYWORDS craniospinal hypovolemia; spinal CSF venous fistula; inferior vena cava obstruction; spine; vascular disorders

A CSF leak in craniospinal hypovolemia (CSH) generally occurs through a spinal dural defect. ${ }^{3,8}$ Despite extensive investigations, quite frequently a dural defect is not identified. ${ }^{13}$ In recent years a spinal CSF venous fistula (SCVF) has been identified as a mechanism of CSF leakage in some of these patients. ${ }^{2,4,10} \mathrm{We}$ report a hitherto undescribed variant mechanism of $\mathrm{CSH}$ that further substantiates that SCVF is an underrecognized mechanism of $\mathrm{CSH}$.

\section{Case Report}

A 57-year-old man was evaluated for a 10 -year history of progressive imbalance, cognitive difficulties, and disabling hypersomnolence. Additional symptoms included a 6-year history of intermittent binocular diplopia, drooling, and dysphagia. He had been on long-term anticoagulation therapy because of recurrent lower-limb deep venous thrombosis. A frontotemporal dementia-like syndrome had been entertained, but sequential brain MRI sequences had shown progressive findings of CSH (tonsillar descent, crowding of the posterior fossa, brainstem distortion with flattening of the ventral pons, and effacement of the basal cisterns) (Fig. 1). The results of MR angiography and MR venography studies of the brain were unremarkable.
A blood patch procedure performed 6 years prior did not improve any of the symptoms. Hypersomnolence and inattention limited what appeared to be a nonfocal neurological examination. The patient had lower-limb hyperreflexia with an extensor plantar response. His calves were prominent and had superficial varicosities. Superficial abdominal varicosities were also present.

Spine MRI revealed a central intramedullary syrinx with possible edema from $\mathrm{C} 1$ to $\mathrm{C} 5$, probably related to compression at the craniocervical junction (Fig. 1). A poorly visualized inferior vena cava (IVC) on the axial cuts of the lower spine MR images raised concern about IVC obstruction. CT venography of the abdomen and pelvis showed a diminutive infrahepatic IVC and chronic thrombotic changes involving bilateral common and external iliac veins (Fig. 2). Prominent vessels seen on the spinal cord surface and within the abdomen and pelvis were suspected to represent collaterals resulting from an obstructed IVC. A CT myelogram showed no CSF leak or SCVF on either immediate or delayed (4 hours) imaging. The following day, multilevel anterior epidural blood patches were administered under CT fluoroscopic guidance, with no demonstrable clinical benefit. Five weeks later, positivepressure intrathecal combined iodinated and gadolinium contrast $\mathrm{CT}$ and MR myelography were performed under 

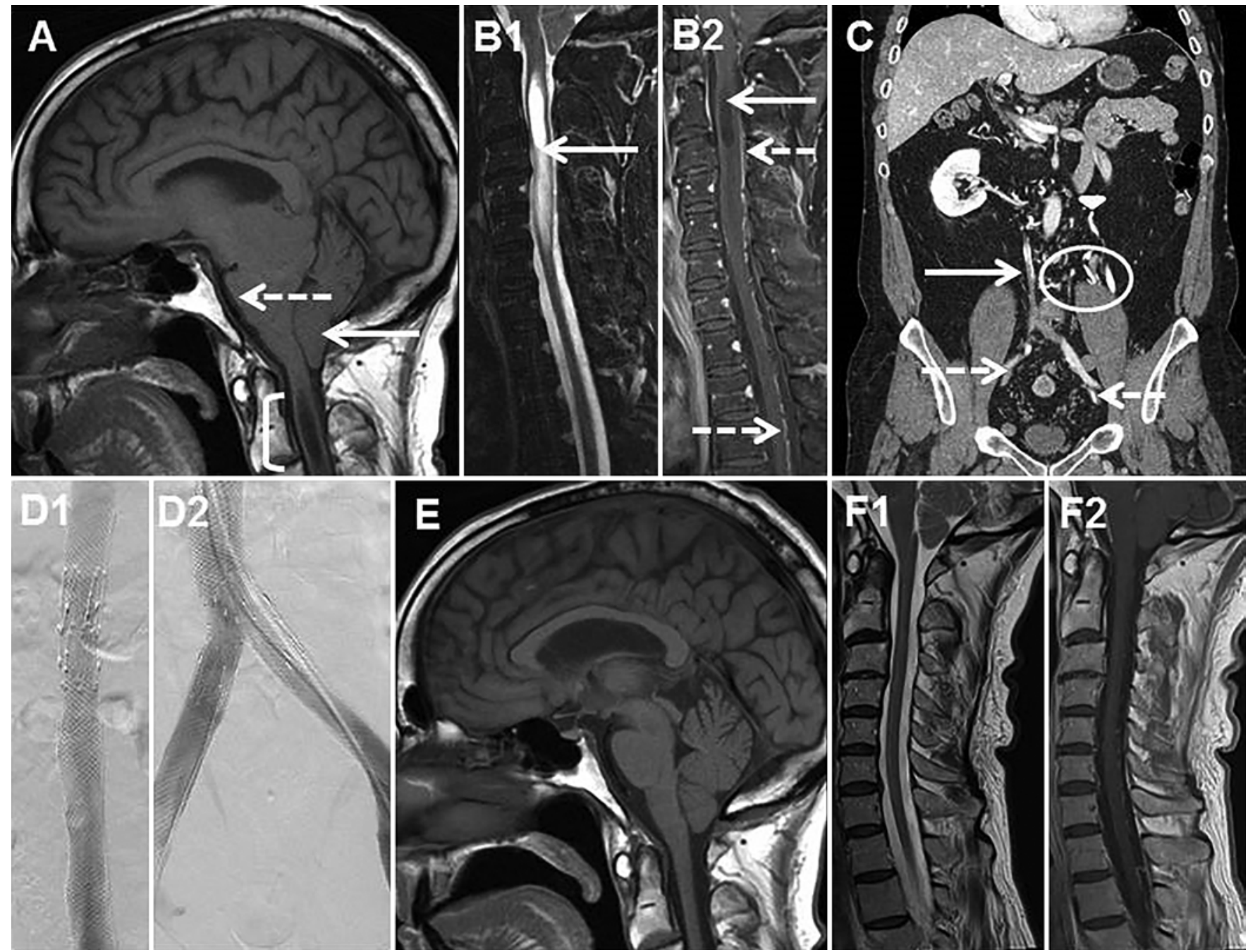

FIG. 1. A: Sagittal T1-weighted brain MRI shows tonsillar descent (arrow), flattening of the ventral pons (dotted arrow), and the upper part of the cervical syrinx/edema (bracket). B1 and B2: Sagittal T2-weighted (B1) and contrast-enhanced sagittal T1-weighted (B2) cervical spine MRI shows intramedullary T2 hyperintensity suggestive of a syrinx/edema (arrows, B1 and B2) and prominent dorsal pial vascularity (dotted arrows, B2). C: Coronal reformatted CT venogram of the abdomen and pelvis shows the diminutive IVC (arrow) and external iliac veins (dotted arrows). The IVC and left external iliac vein show wall calcification also. Multiple curvilinear collaterals are seen (oval). D1 and D2: Venogram shows correction of the IVC (D1) and iliac (D2) stenosis immediately after stenting. E: Sagittal T1-weighted MRI study performed 15 weeks after IVC recanalization shows resolution of imaging evidence of craniospinal hypovolemia. F1 and F2: Sagittal T2-weighted (F1) and contrast-enhanced sagittal T1-weighted (F2) cervical spine $\mathrm{MRI}$ shows resolution of the syrinx and prominent dorsal pial vascularity.

general anesthesia (both with immediate and delayed imaging) but again no leak or SCVF was identified.

The following day, the patient underwent an IVC and bilateral iliac vein recanalization and stenting procedure, which was followed over a few days by prompt and complete resolution of all symptoms. A brain and spine MRI study done 15 weeks later showed resolution of the brain sag, cervical syrinx, and pial vascularity (Fig. 1). A Doppler ultrasound confirmed patency of the IVC and iliac venous stents. Anticoagulation therapy was continued in the same dose. At 15 weeks the dramatic clinical improvement was enduring. An additional follow-up visit 7 months later noted that the patient remained asymptomatic, with normal findings on neurological examination.

\section{Discussion}

Acephalalgic forms of intracranial hypotension, including those with prominent neurobehavioral manifestations, are well recognized. ${ }^{8,13}$ As in our patient, a clinical picture of frontotemporal dementia with neuroimaging evidence of CSH has been described and referred to as frontotemporal brain sagging syndrome..$^{13}$

Investigations done to identify a source of CSF leakage include radionuclide cisternography, CT, and MR myelography (including positive-pressure studies). ${ }^{6}$ The dural defect and resulting CSF leak may be related to a spiculated osteophyte. ${ }^{11}$ At times no source of leakage is identified, and empirical single- or multilevel blood patches are at- 


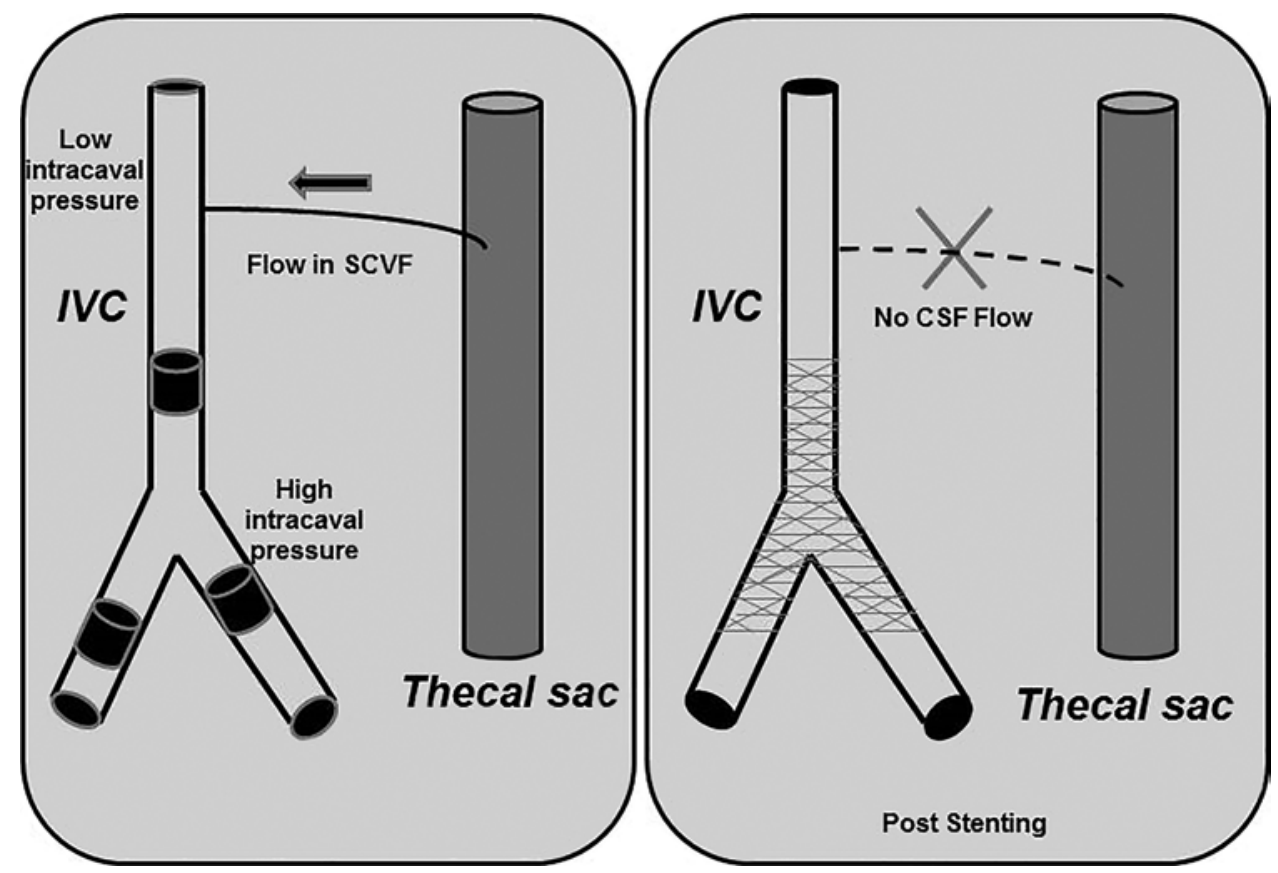

FIG. 2. Proposed mechanism of SCVF. Left: Decreased venous return from IVC stenosis results in low venous pressure in the IVC segment between the stenotic segment and the heart. The pressure differential causes CSF runoff from the thecal sac to the IVC. Right: Following stenting there is normalization of pressure in the IVC segment. This results in a lack of pressure gradient, which renders the SCVF nonfunctional.

tempted. In recent years an SCVF has been identified as a mechanism of CSF leakage without a defect in the dura mater. ${ }^{2,4,10}$

It has been suggested that iatrogenic SCVF may develop during myelography, and intravascular Pantopaque infusion during myelography is probably due to SCVF.5,7 The existence of spinal arachnoid villi and their morphological association with radicular veins has been shown by immunohistochemical studies. ${ }^{12}$

In our case the mechanism of CSH was probably related to an SCVF communicating between the thecal sac and patent infracardiac segment of the IVC, above the chronically occluded infrahepatic IVC. We suspect that the decreased venous return due to the infrahepatic IVC occlusion and the sump effect from the cardiac cycle caused decreased venous pressure in this segment, which in turn yielded a pressure differential between the intrathecal CSF and infracardiac IVC. This pressure differential presumably resulted in development (or perhaps opening) of an SCVF leading to CSF runoff into a perispinal venous collateral and CSH. This proposed pathophysiological mechanism was promptly reversed by stenting, which mitigated the pressure differential, causing functional closure of the fistulae, with subsequent normalization of intrathecal pressure (Fig. 2).

The best-characterized symptoms of IVC and iliac vein occlusion are the so-called postthrombotic syndromes that typically present with chronic leg swelling, venous claudication, and at times chronic lower-extremity venous wounds. Recanalization and stenting of the chronically occluded IVC and iliac veins is a well-recognized procedure with good long-term clinical outcomes. ${ }^{9}$ Chronic
IVC thrombosis may rarely cause a venous congestive myelopathy that can be treated by endovascular stenting. ${ }^{1}$

\section{Conclusions}

This case further supports SCVF as an etiology of dural defect-negative CSH. SCVF should be considered when CSH is accompanied by IVC occlusive disease. Targeted endovascular obliteration of SCVF could be a therapeutic option for cases of CSH that are unaccompanied by a dural defect-related CSF leak.

\section{References}

1. Carvalho DZ, Hughes JD, Liebo GB, Bendel EC, Bjarnason H, Klaas JP: Venous congestive myelopathy due to chronic inferior vena cava thrombosis treated with endovascular stenting: case report and review of the literature. J Vasc Interv Neurol 8:49-53, 2015

2. Clark MS, Diehn FE, Verdoorn JT, Lehman VT, Liebo GB, Morris JM, et al: Prevalence of hyperdense paraspinal vein sign in patients with spontaneous intracranial hypotension without dural CSF leak on standard CT myelography. Diagn Interv Radiol 24:54-59, 2018

3. Kumar N: Beyond superficial siderosis: introducing "duropathies.” Neurology 78:1992-1999, 2012

4. Kumar N, Diehn FE, Carr CM, Verdoorn JT, Garza I, Luetmer PH, et al: Spinal CSF venous fistula: A treatable etiology for CSF leaks in craniospinal hypovolemia. Neurology 86:2310-2312, 2016

5. Lin PM, Clarke J: Spinal fluid-venous fistula: a mechanism for intravascular Pantopaque infusion during myelography. Report of two cases. J Neurosurg 41:773-776, 1974

6. Luetmer PH, Schwartz KM, Eckel LJ, Hunt CH, Carter RE, Diehn FE: When should I do dynamic CT myelography? 
Predicting fast spinal CSF leaks in patients with spontaneous intracranial hypotension. AJNR Am J Neuroradiol 33:690-694, 2012

7. Maillot C: [The space surrounding the spinal cord. Constitution, organization and relationship with the cerebrospinal fluid.] J Radiol 71:539-547, 1990 (Fr)

8. Mokri B: Spontaneous intracranial hypotension. Continuum (Minneap Minn) 21 (4 Headache):1086-1108, 2015

9. Murphy EH, Johns B, Varney E, Buck W, Jayaraj A, Raju S: Deep venous thrombosis associated with caval extension of iliac stents. J Vasc Surg Venous Lymphat Disord 5:8-17, 2017

10. Schievink WI, Moser FG, Maya MM: CSF-venous fistula in spontaneous intracranial hypotension. Neurology 83:472473, 2014

11. Thielen KR, Sillery JC, Morris JM, Hoxworth JM, Diehn FE, Wald JT, et al: Ultrafast dynamic computed tomography myelography for the precise identification of high-flow cerebrospinal fluid leaks caused by spiculated spinal osteophytes. J Neurosurg Spine 22:324-331, 2015

12. Tubbs RS, Hansasuta A, Stetler W, Kelly DR, Blevins D, Humphrey R, et al: Human spinal arachnoid villi revisited: immunohistological study and review of the literature. J Neurosurg Spine 7:328-331, 2007
13. Wicklund MR, Mokri B, Drubach DA, Boeve BF, Parisi JE, Josephs KA: Frontotemporal brain sagging syndrome: an SIH-like presentation mimicking FTD. Neurology 76:13771382,2011

\section{Disclosures}

The authors report no conflict of interest concerning the materials or methods used in this study or the findings specified in this paper.

\section{Author Contributions}

Conception and design: Kumar. Acquisition of data: Neidert, Morris. Analysis and interpretation of data: Neidert. Drafting the article: Kumar. Critically revising the article: Campeau, Morris. Approved the final version of the manuscript on behalf of all authors: Kumar. Study supervision: Kumar, Diehn, Bjarnason.

\section{Correspondence}

Neeraj Kumar: Mayo Clinic, Rochester, MN. kumar.neeraj@ mayo.edu. 